\title{
Extremely Rare Case of an Ectopic Thyroid Tissue Located in the RVOT
}

\author{
Marina Ereva ${ }^{1}$, Georgi Manchev ${ }^{2}$ and Vladimir Danov ${ }^{2}$ \\ 1. Faculty of Medicine, Sofia University "St. Kliment Ohridski”, Sofia 1407, Bulgaria \\ 2. Department of Cardiac Surgery, City Hospital St. Anna, Sofia 1709, Bulgaria
}

\begin{abstract}
INTRODUCTION: We report a case of ectopic thyroid tissue of the heart (struma cordis), which is an exceptionally rare defect in the embryonic development of the primitive gut tube. The most common location in the heart is the right side of the ventricular septum and the right ventricular outflow tract (RVOT). METHOD: The patient is 57 years old woman, presenting with symptoms of heart failure and a systolic murmur best heard at second left intercostal space. The patient had right ventricular hypertrophy measuring $9 \mathrm{~mm}$ in thickness. HISTOLOGICAL DIAGNOSIS: It confirmed differentiated thyroid tissue in the tumor-macro and microfollicular goiter with cholesterol deposits and PAS-positive for colloid. CONCLUSION: First of all we excluded metastatic thyroid carcinoma. Because of the lack of history of deep venous thrombosis and the location of the tumor in an area with high rate of the blood flow, we ruled out the possibility of intraventricular thrombus and suggested neoplasm.
\end{abstract}

Key words: Struma cordis, right ventricle outflow tract, thyroid cancer.

\section{Introduction}

We report a case of ectopic thyroid tissue of the heart (struma cordis), which is an exceptionally rare defect in the embryonic development of the primitive gut tube. The most common location in the heart is the right side of the ventricular septum and the right ventricular outflow tract (RVOT) [1]. Extirpation of the tumor can be performed under total cardiopulmonary bypass (CBP).

\section{Diagnosis}

The patient is 57 years old woman, presenting with symptoms of heart failure and a systolic murmur best heard at the second left intercostal space. Laboratory parameters are normal. ECG shows sinus rhythm and incomplete RBBB. Echocardiography visualized enlarged right heart cavities and an obstructing mass in the RVOT sized $30 / 30 \mathrm{~mm}$ with a peak gradient of $55 \mathrm{mmHg}$ and turbulent flow. The patient had right ventricular hypertrophy measuring $9 \mathrm{~mm}$ in

Corresponding author: Marina Ereva, research field: cardiac surgery. thickness.

\subsection{Differential Diagnosis}

First of all we excluded metastatic thyroid carcinoma. Because of the lack of history of deep venous thrombosis and the location of the tumor in an area with high rate of the blood flow, we ruled out the possibility of intraventricular thrombus and suggested neoplasm.

Extirpation of the tumor was implemented through median sternotomy under total CPB. Surgical access was right paraseptal. A tumor with rubber consistency was found (Fig. 1). It was by a stalk to the front wall the right ventricle (Figs. 2 and 3). The formation was completely removed and the ventriculotomy was closed primarily, without patch, to avoid narrowing of the RVOT (Fig. 4).

\subsection{Histological Diagnosis}

It confirmed differentiated thyroid tissue in the tumor-macro and microfollicular goiter with cholesterol deposits and PAS-positive for colloid (Fig. 5). 


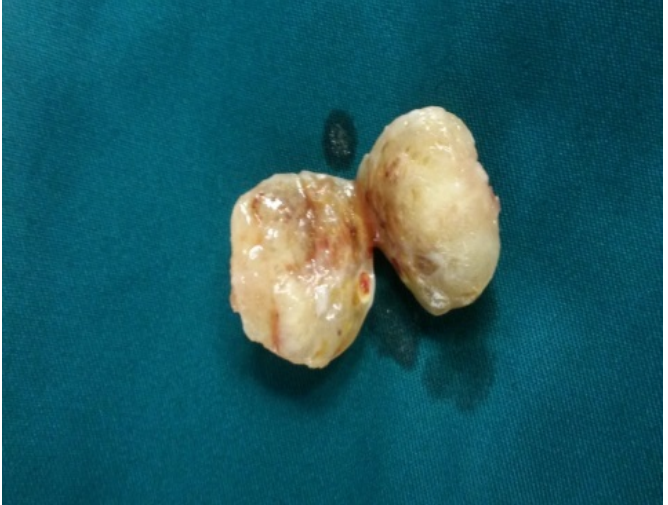

Fig. 1 The removed tumor.

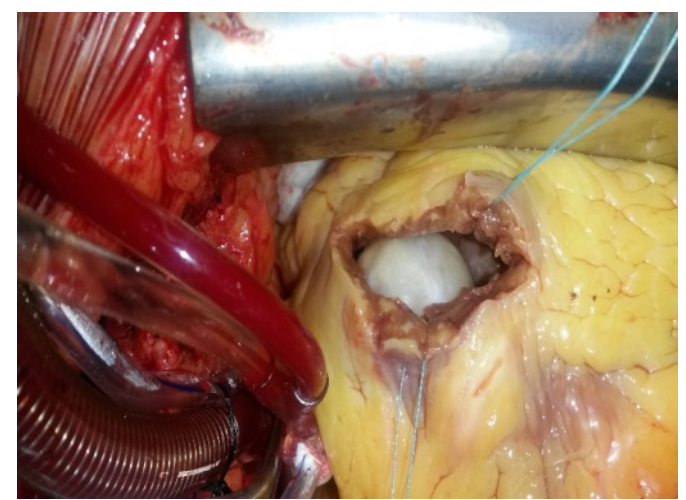

Fig. 2 Tumor attached to the wall.

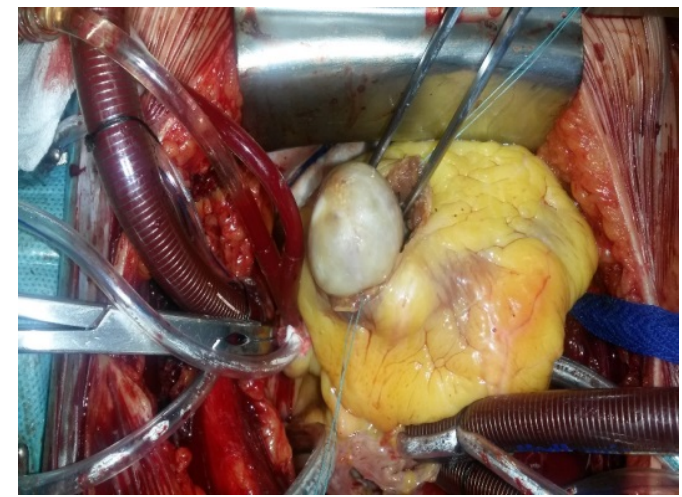

Fig. 3 Removing the tumor.

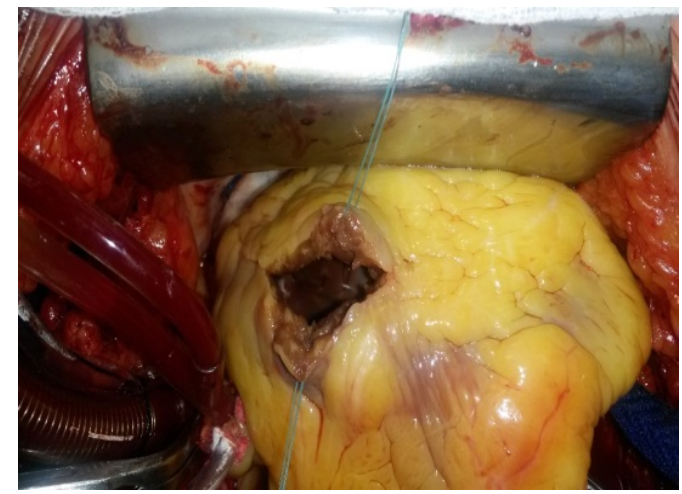

Fig. 4 Closing the ventriculotomy.

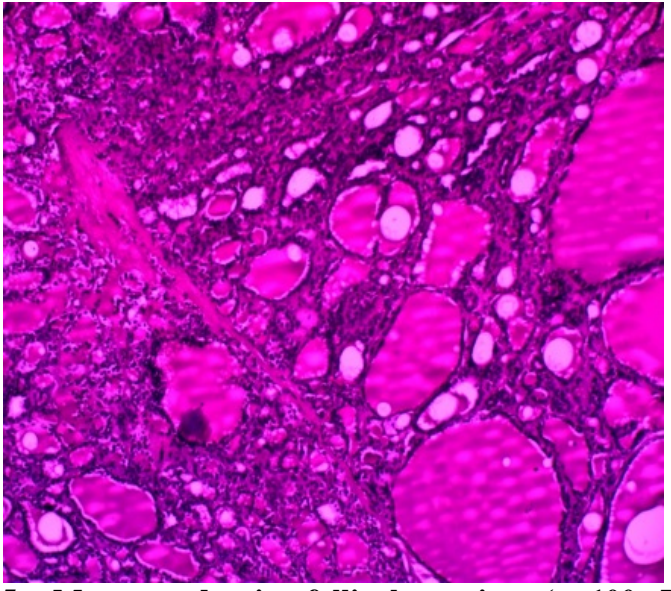

Fig. 5 Macro and microfollicular goiter $(\times 100, \mathrm{H} \& \mathrm{E}$ staining).

The basal membranes were not infiltrated by cells. Consequently, the results point to ectopic thyroid tissue and exclude metastatic thyroid carcinoma.

\section{Discussion}

The most common primary tumour of the heart is left atrial myxoma. The primary thyroid tumor (struma cordis) is one of the rarest tumors of the heart. It is due to abnormal embryogenesis and persistent contact between the thyroid gland primordium and bulbus cordis [2]. More common is metastatic thyroid cancer. Ectopic thyroid tissue can be found in another organs, such as the tongue, cervical lymph nodes, larynx, trachea, mediastinum and diaphragm and it is present there from birth. There is no histological difference between thyroid tissue in a normal position and struma cordis.

\section{Conclusion}

The discovery of tumour in the RVOT should always raise doubt about ectopic thyroid tissue. This type of tumor can be treated conservatively or surgically, but it is important to rule out malignancy [3]. Our patient is a classic example of struma cordis-middle-aged person with symptoms of right heart failure due to obstruction in the RVOT. The location of the tumour is also typical. However, other primary and secondary neoplasms of the heart should be excluded. 


\section{References}

[1] Peng, Ed., Oxenham, H., Foley, M., and Goodwin, A. 2013. "Right Ventricular Outflow Tract Tumor: An Unsuspected Intracardiac Ectopic Thyroid Mass.” Interactive CardVasc. Thor. Surg. 17 (5): 903-5.
[2] Rogers, W. M., and Kesten, H. D. 1962. "Embryologic Basis for Thyroid Tissue in the Heart." Anat. Rec. 142: 323.

[3] Hirnle, T., Szymczak, J., and Ziolkowski, P. 1997. "Ectopic Thyroid Malignancy in the Right Ventricle of the Heart.” Eur. J. CardThor Surg. 12 (1): 147-9. 